\title{
USE OF LOCATION AIDED ALGORITHM USING WI-FI IN INDOOR AD-HOC SYSTEMS
}

\author{
Sivaramakrishnan SR ${ }^{1}$, Gururaja H.S. ${ }^{2}$ \\ ${ }^{1}$ Dept. of Information Science \& Engineering, BMS College of Engineering, Bangalore, Karnataka, India, \\ shivramkrishnan@live.com \\ ${ }^{2}$ Asst. Professor, Dept. of Information Science \& Engineering, BMS College of Engineering, Bangalore, Karnataka, \\ India, gururaja.hs@gmail.com
}

\begin{abstract}
Mobile Ad-Hoc networks are designed to establish a network anytime and anywhere. Unlike other networks, ad-hoc does not require a fixed infrastructure. There are various routing techniques which are related to ad-hoc networking like Destination Sequenced Distance Vector Protocol (DSDV), Dynamic Source Routing Protocol (DSR) and Ad-Hoc On Demand Distance Vector Protocol (AODV). One such routing protocol named Location Aided Routing uses GPS (Global Positioning System) to get the coordinates of the nodes which would assist in routing. There is a definite set of drawbacks where GPS is used to get the location for the nodes in the ad-hoc network which is implemented indoor. In this paper, we propose an alternative of using Wi-Fi Positioning System to get the location of the co-ordinates which may prove to be a better solution than GPS indoor.
\end{abstract}

Index Terms: Ad-Hoc Networks, DSDV, DSR, AODV, LAR, GPS, Wi-Fi Positioning System

\section{INTRODUCTION}

Mobile Ad-Hoc networks (MANET) are a collection of mobile nodes with a dynamic network infrastructure which forms a temporary network. The topology of ad-hoc networks can change quickly; the network should be adaptable to changes including when a node leaves the network or when a new node joins the network. Therefore, the task of routing in MANET cannot be considered trivial. There are several efficient protocols which can be implemented on MANET. These protocols can be roughly classified as either a Table-Driven Protocol (Proactive Protocol) or a Source-Initiated Protocol (Reactive Protocol) [1]. In this paper, we closely look into Location Aided Routing which uses location co-ordinates to decrease the overhead of route discovery. LAR uses GPS to obtain the co-ordinates of the various nodes in the network. Using these co-ordinates the expected zone and also the request zones are defined. GPS brings in an overhead when used. The main limitation of GPS is that it is not accurate when used indoors. Therefore it may not be effective in the case of ad-hoc networks using LAR, which are implemented indoors. In this paper, we provide an alternative to GPS, which is Wi-Fi Positioning System to get the co-ordinates of the nodes in the ad-hoc network implemented indoors.

\section{FLOODING}

Flooding is a simple routing algorithm where a packet is broadcasted to every link other that the link from which the packet arrived from. When a Node A needs to find a route to Node G, Node A issues a Route Request message to all its neighbors. Every node in the network is given a unique identifier. The Route Request message contains the identifier of the destination node. Suppose an intermediate Node $E$ receives the route request message, it compares its identifier with that of the destination present in the message. If it is a match then the request for a route is to itself. If not, Node E broadcasts the message to its neighbors. Redundancy is avoided as the Node B would discard the same Route Request from Node A, when received.

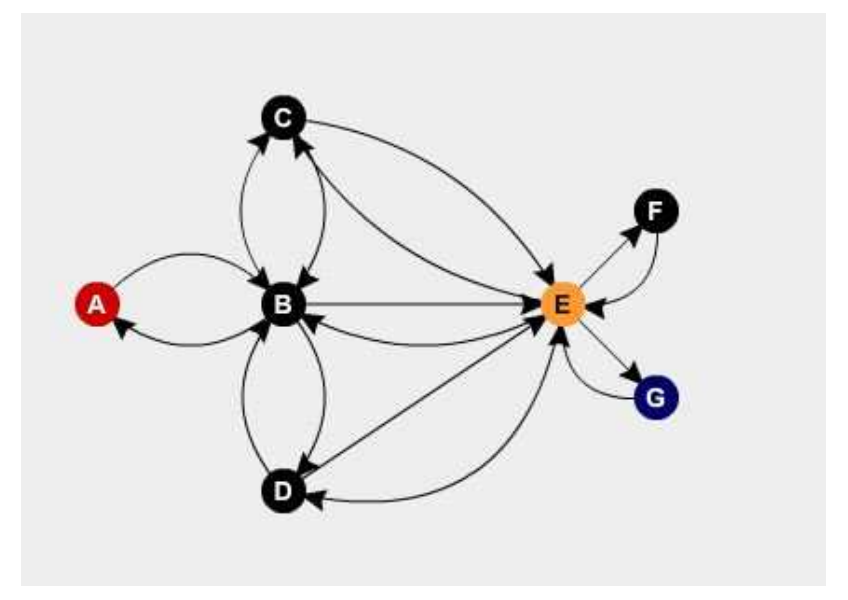

Fig - 1: Demonstration of Flooding

One receiving the Route Request message, Node G sends a Route Reply message to Node A. The path is obtained by reversing the path obtained from route request message. Dynamic source routing (DSR) [2], and ad-hoc on demand Distance vector routing (AODV) [3] protocols are both based on variations of flooding. 


\section{OVERVIEW OF LOCATION AIDED ROUTING}

Flooding is not an efficient algorithm, though it may be the simplest to implement. A lot routing overhead is involved in the case of Flooding, as the packets are broadcasted to all. An alternative to Flooding is Location Aided Routing (LAR). Location Aided Routing uses the Global Positioning system (GPS) to obtain the physical location of the nodes which is used to reduce the overhead involved. LAR uses two different types of region - Expected Zone and Request Zone.

\section{Expected Zone}

Consider a situation where the Node A needs to find a route to Node B. Assume that, Node A knows the location of Node $B$ at time $t_{0}$ and the average speed with which the node $\mathrm{B}$ is moving. Let the average speed be $\mathrm{v}$. Expected Zone is defined as the region in which the Node A expects the Node $B$ to be present at time $t_{1}$ where $t_{1}>t_{0}$. Expected Zone can be defined as a circle with a radius of $v\left(t_{1}-t_{0}\right)$.

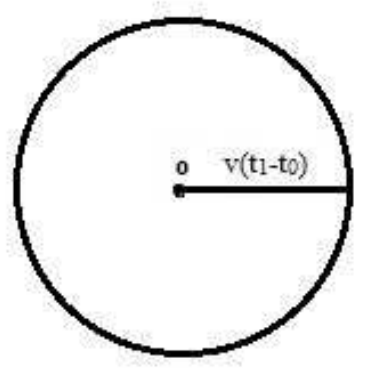

Fig - 2: Example of Expected Zone with radius v $\left(\mathrm{t}_{1}-\mathrm{t}_{0}\right)$

\section{Request Zone}

In Flooding, each node broadcasts its route request packet to its neighbors. But this is not the case in LAR. A region known as Request Zone is defined. A Node would broadcast the message to its neighbors only if it is in the request zone.

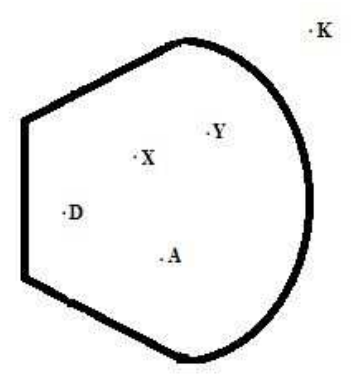

Fig - 3: Example of Request Zone

If Node $\mathrm{X}$ receives route request message, it checks if it lies within the Request Zone. Since it is present inside the Request Zone, Node $\mathrm{X}$ broadcasts its message to its neighbors. But this is not the case with Node K. Since node $\mathrm{K}$ is present outside the Request Zone, it discards the Route Request packet which it received.
Using the Expected Zone and Request Zone, there are two LAR Schemes defined [4].

\subsection{LAR Scheme 1}

In this is scheme it is assumed that the source Node $\mathrm{S}$ knows the co-ordinates of destination Node $\mathrm{D}$ at time $\mathrm{t}_{\mathrm{o}}$ and also its average velocity at which it's moving. The Request Zone is defined as the smallest rectangle which contains the Source Node $\mathrm{S}$ and the Expected Zone such that the sides of the rectangle are parallel to the $\mathrm{X}$ and $\mathrm{Y}$ axis. The source $\mathrm{S}$ in its Route Request message includes the co-ordinates of the four corners of the rectangle. When an intermediate node receives this route request, it compares its location with that of the request zone. It will discard the message if it lies outside the expected zone, otherwise it would forward the route request message to its neighbors.

In the following example, when Node I receives the Route Request message from Node $\mathrm{S}$, it discard the message because Node I is present outside the Request Zone.

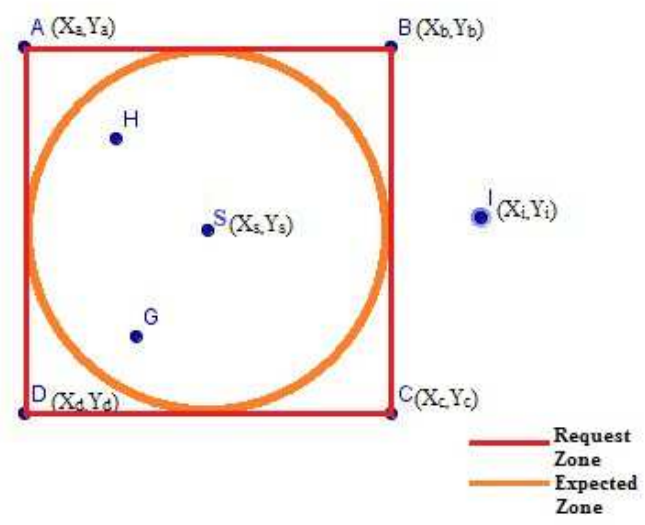

Fig - 4: LAR Scheme 1

\subsection{LAR Scheme 2}

In addition to the location of the request zone in the route request message, LAR Scheme 2 also includes the distance (between the Source Node S and Destination Node D) and the co-ordinates of the destination. The distance between the source and the destination is denoted by DIST $\mathrm{s}_{\mathrm{s}}$ and the coordinates of the destination are represented by $\left(\mathrm{X}_{\mathrm{d}}, \mathrm{Yd}\right)$. When an intermediate node I receives the route request from the Source, it calculates its distance from the destination denoted by DIST $_{\mathrm{i}}$. For some parameter $\delta$, DIST $_{\mathrm{s}}+\delta \geq$ $\mathrm{DIST}_{\mathrm{i}}$, the Node I would forward the route request message to its neighbors. 


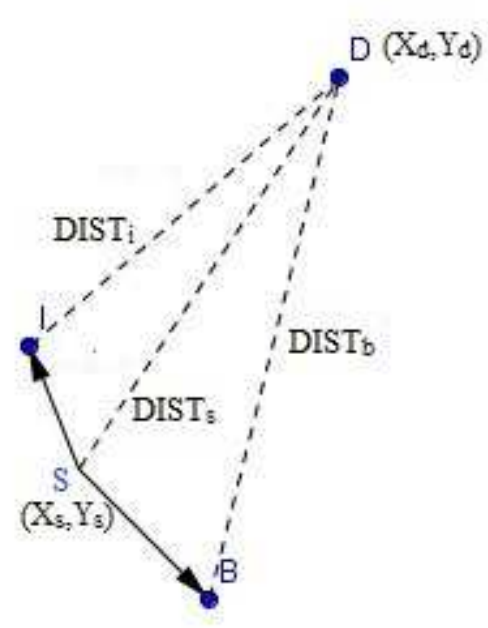

Fig - 5: LAR Scheme 2

The distance between node $\mathrm{B}$ and the destination is denoted by $\operatorname{DIST}_{\mathrm{b}}$. But $\mathrm{DIST}_{\mathrm{s}}+\delta \leq \mathrm{DIST}_{\mathrm{b}}$, therefore the Node B discards the route request packet.

\section{WI-FI OVER GLOBAL POSITIONING SYSTEM}

The key components of the Location Aided Routing are the co-ordinates of the nodes. These co-ordinates could be obtained in many ways including, Global Positioning System and Wi-Fi Positioning Systems. The Global Positioning System (GPS) is a network of satellites orbiting the Earth at an altitude of 20,000 km. The U.S Government designed this system for military purpose, but now it can be used by anyone who has a GPS enabled system. At least four GPS satellites are visible at any time, wherever you are on the planet. Each satellite transmits information about its position and the current time at regular intervals. These signals, travelling at the speed of light, are intercepted by your GPS receiver, which calculates how far away each satellite is based on how long it took for the messages to arrive. Using the process of trilalteration, the exact location could be pin pointed by the GPS receiver.

There is a considerable error involved while using the GPS. According to the Official U.S Government Information about the GPS, the errors associated with GPS (in terms of location) are about 7.8 meters at $95 \%$ confidence. [5] Also, GPS faces a problem of Satellite Geometry. If a GPS receiver is locked onto four satellites and all four of these satellites are in the sky to the north and west of the receiver, satellite geometry is rather poor. [6] Considering all the above factors, GPS does have its limitations. By using GPS in ad-hoc networks implemented indoors, these factors play a crucial role. The energy consumed by the GPS to obtain the co-ordinates would be significant. Since ad-hoc networks are designed to last for a long duration, GPS may prove to be in efficient. Energy Efficient protocols for adhoc networks such as SPAN [7] have been developed which builds on the observation that only certain nodes needs to be active at a time to forward traffic.

When ad-hoc systems using LAR is implemented indoors, or in a densely populated location with a number of buildings, the GPS may also not be accurate due to multipath. Multipath occurs when the signal bounces off a building before reaching the GPS receiver's antenna which causes a delay in the signal. This added time makes the GPS receiver think the satellite is farther away than it really is.

In this paper we propose an alternate method of obtaining the co-ordinates of the nodes in the Location Aided Routing (implemented indoors) with the help of Wi-Fi Positioning System instead of Global Positioning System. The mean error involved with Wi-Fi Positioning System is found to be about $2.3 \mathrm{~m}$ (line of sight) and 2.9(non-line of sight) [8]. This is comparatively lesser than the error generated in GPS. Also the limitations such as multipath is avoided in this system

\section{WI-FI AS AN ALTERNATIVE}

Wi-Fi technology enables electronic devices to exchange data or connect to the internet wirelessly. It is based on the Institute of Electrical and Electronics Engineers (IEEE) 802.11 standards (which are protocols to implement WLAN) [9].Many devices can access Wi-Fi. Devices can connect to a network resource such as the internet using various wireless access points. Wi-Fi positioning system uses these wireless access points to measure the intensity of the signal. There are different methods by which the signal strength or the direction of the signal could be used to determine the location of the node.

\subsection{Cell Identity}

In order to find the location of the node through this method, some preparations must be made. A database of wireless access point's IDs (Which could be the MAC Address) and its geo-location is necessary. The closest station to the node is the station from which the strongest incoming signal on the node is received. A request to the database is performed for the ID of the station with the strongest received signal. The database returns the position of the desired station and from this the location of the node could be obtained. But this system is found to be very inaccurate and can be used only to find the approximate location of the node. This is because it uses the location of only one wireless access point and the request to the pre defined database would return an approximate location close to that wireless access point. 


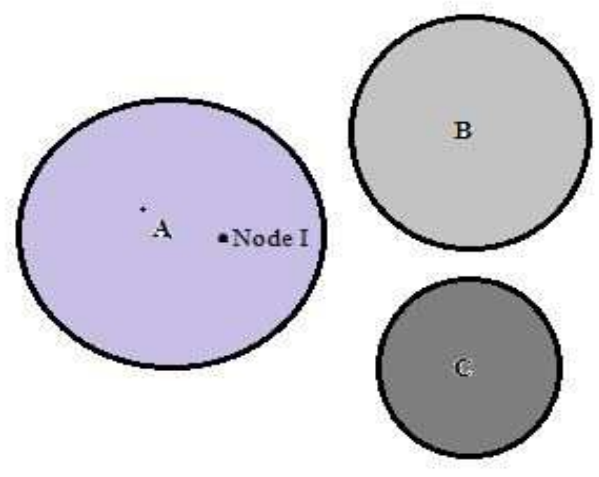

Fig - 6: Example of Cell Identity

Node I is receiving the strongest strength from wireless access point $\mathrm{A}$. On sending a request to the database with the ID of wireless access point, it returns an approximate location of Node I.

\subsection{Trilateration}

Trilateration[10] is the process of determining the location of a node by measuring distances from three different wireless access points. This process starts off by calculating the distance between the node and the first wireless access point. Let the distance obtained be denoted by $\mathrm{WD}_{1}$. This distance obtained is used to define a circular region with radius of $\mathrm{WD}_{1}$ from the first wireless access point. In other words, the node lies within this circle of radius $\mathrm{WD}_{1}$. To increase precision, another wireless access point is considered. The distance between the node and the second wireless access point is calculated and let it be denoted by $\mathrm{WD}_{2}$. This distance of $\mathrm{WD}_{2}$ is used to define a circular region with the radius of $\mathrm{WD}_{2}$ with the second wireless access point as the centre. Now there are two circles with radius's $\mathrm{WD}_{1}$ and $\mathrm{WD}_{2}$ which are intersecting at two points (one of them being the location of the node). In order to pin point on the exact location, a third wireless access point is considered. The distance between the third wireless access point and the node is calculated and is represented by $\mathrm{WD}_{3}$. A third circle is drawn with the wireless access point 3 as the center and $\mathrm{WD}_{3}$ being the radius. The intersection of the three circles is the location of the Node. An illustration of Trilateration is shown below.

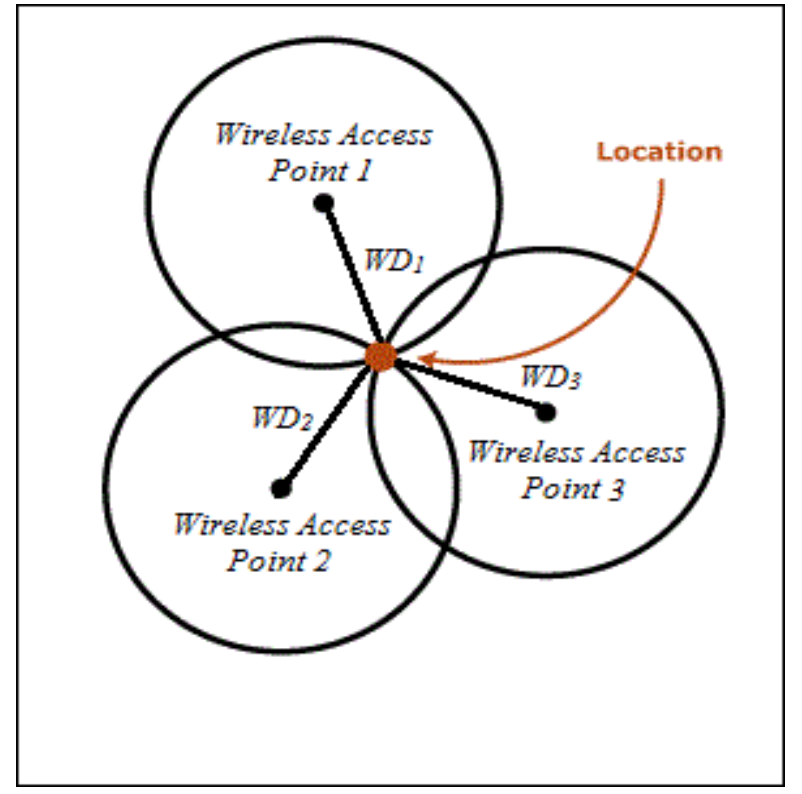

Fig - 7: Trilateration

\subsection{Wi-Fi Fingerprinting}

Wi-Fi Fingerprinting [11] technique involves forming a predefined table of values of signal patterns and this table is referred for obtaining the corresponding location. The initial phase involves recording of the entire area of work. The area should be covered with pattern of recording points known as fingerprints. Every fingerprint consists of measurements including the signal strength of various wireless access points in that location. This data is stored in a database known as radio map. If a node should be located in the recorded area, then it measures the signal strengths of all the current wireless access points in range. This data which is obtained by the node is compared with the radio table to obtain the position of the node. Table 1 shows an example of a Radio Map which is used in the Wi-Fi fingerprinting technique.

Table -1: Example of a Radio Map

\begin{tabular}{|c|c|c|c|c|}
\hline Position & Direction & WAP 1 & WAP 2 & WAP 3 \\
\hline P1 & 0 & $-89 \mathrm{dBm}$ & $-43 \mathrm{dBm}$ & $-12 \mathrm{dBm}$ \\
\hline P2 & $40^{\circ}$ & $-30 \mathrm{dBm}$ & $-98 \mathrm{dBm}$ & $-45 \mathrm{dBm}$ \\
\hline P3 & $55^{\circ}$ & $-20 \mathrm{dBm}$ & $-10 \mathrm{dBm}$ & $-39 \mathrm{dBm}$ \\
\hline P4 & $67^{\circ}$ & $-67 \mathrm{dBm}$ & $-67 \mathrm{dBm}$ & $-12 \mathrm{dBm}$ \\
\hline
\end{tabular}

The strength of the Wi-Fi signal at a particular location is measured in terms of decibel meter $(\mathrm{dBm})$. The three wireless access points which are needed are represented as WAP1, WAP2 and WAP3. Suppose a node is located at a position where the intensity of WAP1, WAP2 and WAP3 are $89 \mathrm{dBm},-43 \mathrm{dBm}$ and $-12 \mathrm{dBm}$ respectively. The node sends a request to the radio map to match these values of WAP1, WAP2 and WAP3. On comparing the values of the radio map and the values sent by the node, the position of the node is found to be in P1. 


\section{CONCLUSIONS}

Wi-Fi in today's world is ubiquitous. By replacing Global Positioning System for obtaining the co-ordinates of nodes for LAR with Wi-Fi Positioning System may prove to be of a greater benefit. Wi-Fi is proven to be faster than GPS in finding out location. The GPS module used to obtain the coordinates will consume considerable amount of power. Unlike GPS, the power consumed by Wi-Fi would be lesser because the location is obtained from the already defined regions of the wireless access point. By using one of the methods mentioned in this paper, we might possibly attain an ad-hoc network which could be implemented indoors with greater energy efficiency. Also if GPS were to be used in LAR indoors, the problem such as multipath would detriment the accuracy of the location. This could be overcome in the case of using Wi-Fi where multipath is minimum. Wi-Fi Positioning System may be a better option in the case of indoor ad-hoc networks than GPS.

\section{REFERENCES}

[1]. Mbarushimana, C and Shahrabi A., "Comparative Study of Reactive and Proactive Routing Protocols Performance in Mobile Ad-Hoc Networks", May ,2007.

[2]. D. Johnson and D. A. Maltz, "Dynamic source routing in ad-hoc wireless networks, Kluwere Academic Publishers, 1996.

[3]. C. E. Perkins and E. M. Royer, "Ad-Hoc on demand distance vector (AODV) routing (Internet-Draft)," Aug. 1998.

[4]. Yottttg-BaeKo and Nitin H. Vaidya, "Location-Aided Routing (LAR) in Mobile Ad-Hoc Networks", 2000.

[5]. "Global Positioning System Standard Positioning Service Performance Standard", by the U.S. Government available at http://www.gps.gov/technical/ps/2008-SPSperformance-standard.pdf.

[6]. C Dussault, R Courtois, JP Ouellet, J Huot, "Influence of satellite geometry and differential correction on GPS location accuracy", Wildlife Society Bulletin, 2001.

[7]. B Chen, K Jamieson, H Balakrishnan, R Morris, "Span: An energy-efficient coordination algorithm for topology maintenance in ad-hoc wireless networks", Wireless networks, 2002 - Springer.

[8]. Bose, A., Chuan Heng Foh, "A practical path loss model for indoor Wi-Fi positioning enhancement", Information, Communications \& Signal Processing, 2007 6th International Conference.

[9]. Crow, B.P; Widjaja, I.; Jeong Geun Kim; Sakai, P.T, "IEEE 802.11 Wireless Local Area Networks", Communications Magazine, IEEE (Volume:35 , Issue: 9).

[10]. F. Lassabe, P. Canalda, P. Chatonnay, F. Spies, "Indoor Wi-Fi positioning: techniques and systems", Springer October 2009, Volume 64, Issue 9-10, pp 651-664. [11]. E. Mok \& G. Retscher, "Location determination using Wi-Fi Fingerprinting vs. Wi-Fi trilateration“, Journal of Location Based Services Volume 1, Issue 2, 2007.

\section{BIOGRAPHIES}

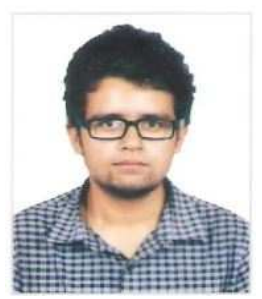

Sivaramakrishnan SR is pursuing his Bachelor of Engineering in Information Science at BMS College of Engineering, Bangalore. His research interests include Adhoc networks, Sensor networks and Network Security.

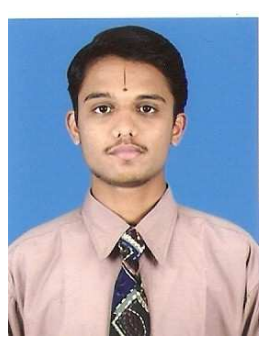

Gururaja H.S. has completed his Bachelor of Engineering in Computer Science and Master of Technology in Computer Networks from Visvesvaraya Technological University, Belgaum. $\mathrm{He}$ is currently pursuing his $\mathrm{Ph}$.D. from JNTU, Hyderabad in the field of Cryptography \& Network Security and has around 8 years of teaching experience. 